\title{
Jet Flapping Control with Acoustic Excitation
}

\author{
Shouichiro Iio ${ }^{1 *}$, Ken Hirashita ${ }^{1}$, Yusuke Katayama ${ }^{1}$, Yoshiaki Haneda ${ }^{2}$, \\ Toshihiko Ikeda ${ }^{1}$, Tomomi Uchiyama ${ }^{3}$ \\ ${ }^{1}$ Department of Environmental Science and Technology, Shinshu University, Nagano, Japan \\ ${ }^{2}$ Department of Mechanical Engineering, Nagano National College of Technology, Nagano, Japan \\ ${ }^{3}$ EcoTopia Science Institute, Nagoya University, Nagoya, Japan \\ Email: ${ }^{*}$ shouiio@shinshu-u.ac.jp
}

Received February 8, 2013; revised April 5, 2013; accepted May 1, 2013

Copyright (C) 2013 Shouichiro Iio et al. This is an open access article distributed under the Creative Commons Attribution License, which permits unrestricted use, distribution, and reproduction in any medium, provided the original work is properly cited.

\begin{abstract}
The dynamics of flapping motion of a rectangular jet under acoustic excitation is studied experimentally by means of hot-wire measurement and flow visualization with smoke method. The excitation sufficiently enables "phase-lock", which permitted us to extract the organized wave motion from a background field of finite turbulent fluctuations. The mean and fluctuation velocity are investigated and focused on the excitation frequency and the Reynolds number. As the excitation frequency decreases, it was found that the jet flapping and the jet spread were enhanced. The excitation with sub-harmonic frequency has significant effects on the rectangular jet behavior. The maximum value of the periodic velocity fluctuation strongly depends on the excitation frequency.
\end{abstract}

Keywords: Jet Control; Rectangular Jet; Vortex; Hot Wire; Visualization

\section{Introduction}

The development of a large coherent structure in a mixing layer of jet flow is responsible for some of the most fascinating aspects of fluid dynamics, such as mixing, transport and instability, etc. In the fields of engineering, jet flows have been extensively investigated because of their widespread applications. The pioneering works on coherent structures in turbulent flow were first confirmed by Crow and Champagne [1] and Brown and Roshko [2]. A lot of researches have been carried out concerning the mechanism and role of coherent structures in jet flows [3-9]. Previous experimental observations by Sato [10] revealed that the jet had sensitivity to external sound, and different initial modes of evolution could lead to distinct growth rates of mixing layers. Ho [11] proposed that the fundamental and sub-harmonic instabilities were significant effects on the vortex formation and merging processes. Ho and Gutmark [12] found that a free jet could be effectively excited by acoustic disturbances in its inherent frequency to obtain more upstream position of a vortex roll-up and more localized, organized and higher energy containing coherent structures. Goldschmidt and Bradshaw [13] originally suggested the flapping motion based on the quasi-periodic negative cross-correlation

"Corresponding author. between the fluctuating longitudinal velocities on either side of the jet centerline. Gortari and Goldshmidt [14] suggested an inviscid mechanism for the flapping motion. Atassi and Lueptow [15] assessed the flapping motion using the linear inviscid analysis and the nonlinear finite-amplitude analysis. Acoustic excitation in this study is one of a zero net mass flux control method. Recently, synthetic jet actuators, which is a net zero mass flux jet, have been shown to be a useful tool for flow control, especially flow vectoring [16] and mixing [17,18]. Jet flow characteristics strongly depend on a forcing condition.

The main objective of the present paper is to examine experimentally the effect of excitation Strouhal number based on the excitation frequency and a mean jet velocity on the jet flapping motion. We conducted flow visualization with smoke method and a velocity measurement to clarify flapping phenomena of the acoustically controlled jet. The flow visualization focused on a development in space of coherent structure evolution in the excited rectangular jet. In order to circumstantially grasp the effects of acoustic excitation on the jet flapping, a concept of phase average was applied to a hot-wire measurement [19]. Since the coherent structure signature and the flapping motion were visually and quantitatively evaluated. 


\section{Experimental Apparatus and Procedure}

A schematic illustration of an experimental setup is shown in Figure 1. The test section including a wind tunnel and a nozzle are set in a sound proof chamber to attenuate perturbations such as noises from outside. Dimensions of the chamber are $2000 \mathrm{~mm}$ in height, 1800 $\mathrm{mm}$ in width and $2700 \mathrm{~mm}$ in length. The inner wall of the chamber is covered with several pieces of glass wool mat of $50 \mathrm{~mm}$ thickness except for the floor. A ceiling of the chamber has a socket to mount of the wind tunnel consisted of a silent duct and a flow-rectifying device such as a honeycomb and wire screens. A contraction nozzle is mounted of the tunnel exit. The side wall of the chamber has an exhaust opening to connect through another silent duct to a blower settling outside of the chamber. The blower generates negative pressure by sucking up air in the chamber, then the jet issues from the nozzle exit. The jet velocity is regulated with a variable speed motor attached to the blower. In this experiment, the background noise is $57.9 \mathrm{~dB}$ for the blower stopping.

The detailed illustration around a test section is shown in Figure 2. The coordinate system is fixed at the nozzle center, the origin is located at the center of the jet exit, the $x$-axis is along the jet flow direction, the $y$-axis is in the horizontal direction perpendicular to the flow, and the $z$-axis is in the spanwise direction of the nozzle. The tunnel has a rectangular cross section nozzle. Dimensions of the nozzle are $10 \mathrm{~mm}$ in width, $w, 150 \mathrm{~mm}$ in length, $h$, the aspect ratio $(h / w)$ of 15 , and the contraction ratio is 10.0. A honeycomb and fine screens are installed in the tunnel for further managing the flow quality. The flow velocity at the origin was set equal to $U_{0}=10,20,30 \mathrm{~m} / \mathrm{s}$ which correspond to Reynolds numbers of $6.7 \times 10^{3}, 1.3 \times$ $10^{4}, 2.0 \times 10^{4}$. The flow velocity, $U_{0}$, is calculated from measured pressure drop across the nozzle. The flow

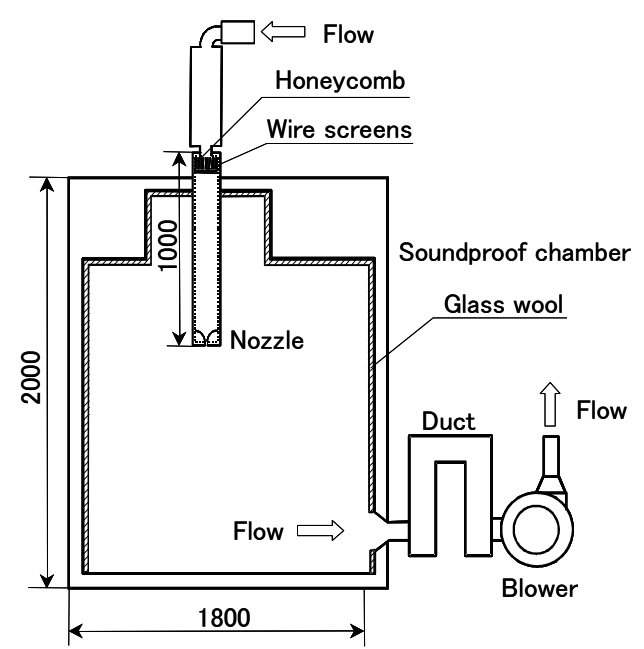

Figure 1. Experimental setup (Unit in $\mathrm{mm}$ ).

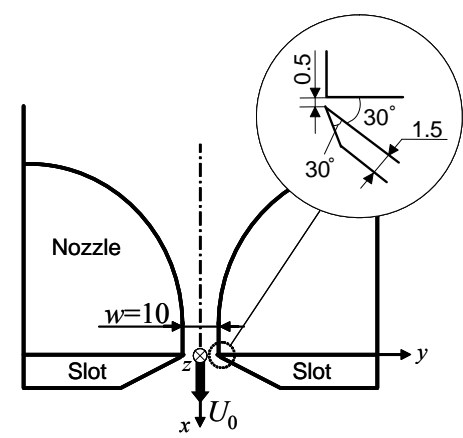

Figure 2. Test section (Unit in $\mathbf{m m}$ ).

temperature is kept constant by an air conditioner and a ceiling fan positioned outside the chamber.

Acoustic disturbance is inserted locally through a pair of slot devices which set at the nozzle exit in the spanwise direction of the nozzle. Excitation amplitude is defined as $u_{p r m s}$ that the root mean square value of fluctuating velocity at the slot exit. $u_{\text {prms }} / U_{0}$ is set at $2.0 \%$. It was confirmed that vortex shedding timing is synchronized with an excitation period at $u_{\text {prms }} / U_{0} \geq 0.1 \%$. In particular, $u_{p r m s} / U_{0}=2 \%$ is the most effective condition for jet flapping control. It is noted that streamwise turbulence intensity at the nozzle exit was about $0.4 \%$ of $U_{0}=10 \mathrm{~m} / \mathrm{s}$. The excitation frequency, $f_{p}$, was set from 50 to $900 \mathrm{~Hz}$ at $50 \mathrm{~Hz}$ interval. The jet is excited with the asymmetrical perturbation. The details of the slot structure are shown in a blow up in Figure 2. An opening gap of the slot tip is $0.5 \mathrm{~mm}$ and the slot meets the nozzle exit plane at an angle of $30^{\circ}$. The slot device is shown in Figure 3. The slot is partitioned into eight cells by thin Plexiglas plates shown as Figure 3, and it is therefore possible to impose either symmetric or asymmetric excitation on the jet exit. The slot is connected to a loudspeaker by vinyl pipes having an inner diameter of $9.5 \mathrm{~mm}$. Power of the loudspeaker is 300 watts and the diameter is $300 \mathrm{~mm}$.

The velocity measurement was performed by using a constant temperature anemometer and a hot-wire probe that consisted of a $2.5 \mu \mathrm{m}$ platinum wire with a length of $1 \mathrm{~mm}$. The hot wire is calibrated by a high-precision pressure transducer and Pitot-tube measurements according to the King's law. A 16-bit A/D converter is connected to a personal computer for data acquisition. The probe is inserted into a flow from a downstream position by a handmade support to attenuate a flow disturbance. The sensing probe is facilitated by a three-dimensional traversing system that is driven by stepping motors under the personal computer controlled.

Flow visualization was performed by making an alcohol mist suck with the inlet air from the upstream of the tunnel. Photographic recordings of the flow visualization were obtained by employing a six-megapixel camera positioned outside the flow. The smoke was illuminated by flashlight through a slit of $1.5 \mathrm{~mm}$ width. 


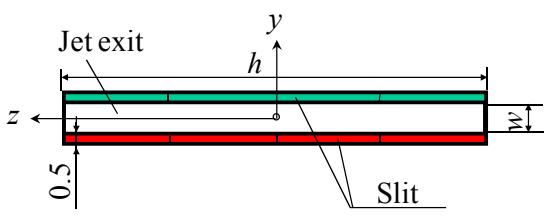

Figure 3. Slot device (Unit in mm).

\section{Phase Average Technique}

The eduction of the coherent structure as well as interpretation of the data requires introduction of the concept of phase average as suggested by Hussain and Reynolds [20]. In this experiment we used the cyclic position of the speaker signal as a clock for selective sampling, which permitted us to extract the organized wave motion from a background field of finite turbulent fluctuations. We decomposed the fluctuating velocity $u(x, t)$ as

$$
u(x, t)=U(x)+\tilde{u}(x, t)+\hat{u}(x, t)
$$

$U$ is the streamwise mean velocity, $\tilde{u}$ is the periodic (phase average) velocity and $\hat{u}$ is the turbulence velocity. We also used the definition of the phase average velocity at a particular time $\theta$ in the period $T$ of the periodic perturbation, then

$$
\tilde{u}(x, \theta)=\lim _{N \rightarrow \infty} \frac{1}{N} \sum_{n=1}^{N}\{u(x, \theta+n T)-U(x)\}
$$

The difference between the instantaneous signal and the phase average represents the background random fluctuation, and the difference between the phase average and the time average denotes the (periodic) coherent component. Thus, knowing the period of the induced perturbation, the mean, coherent and random components of the velocity signal can be extracted from the instantaneous total velocity signal.

\section{Result and Discussion}

\subsection{Jet Flow Visualization}

Initial conditions of a jet are important to development of the coherent structures in a shear layer and to investigate the flapping motion. The fundamental frequency, $f_{0}$, was first measured from frequency spectra along the jet axis to estimate the excitation frequency, $f_{p}$, at $U_{0}=10 \mathrm{~m} / \mathrm{s}$. The power spectra of the velocity fluctuation measured along the jet centerline are shown in Figure 4. The dominant frequency was observed after $x / w=3.5$, its value was $f_{0}=300 \mathrm{~Hz}$, and reached the peak at $x / w=5$, and then decreased. The sub-harmonic frequency was observed in the range $x / w>4.5$. The Strouhal number based on the natural vortex shedding frequency and the nozzle width is $S t=0.30$. The sub-harmonic frequency was gradually appeared at $x / w=4.5$, and reached the peak at $x / w=6.0$, and observed broader band. In the range of 4.5

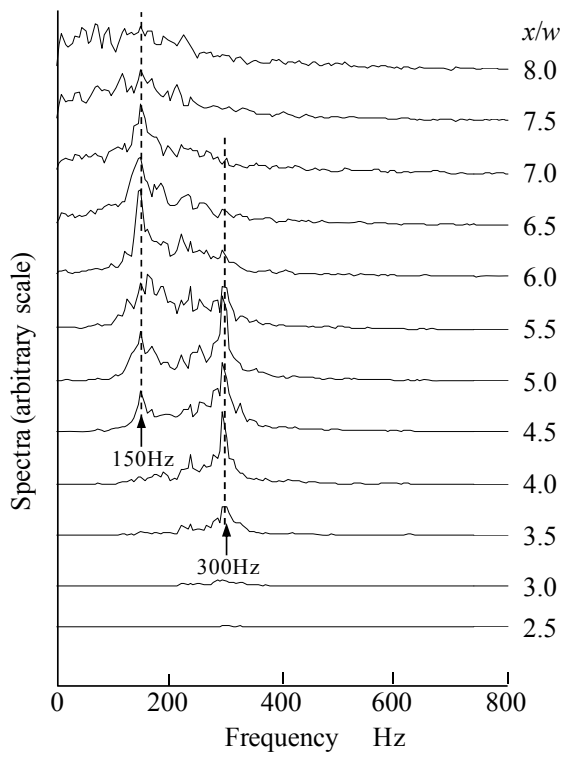

Figure 4. Spectra of velocity fluctuation along jet center line $\left(\operatorname{Re}=6.7 \times 10^{3}\right)$.

$<x / w<5.5$, the both frequency components were clearly appeared. The frequency of a large-scale vortex shedding agrees well with the fundamental frequency, $f_{0}$, of the spectrum. The velocity spectrum enables us to exactly determine the excitation frequency, so the excitation frequency, $f_{p}$, was set to $150\left(=f_{0} / 2\right), 300\left(=f_{0}\right), 550 \mathrm{~Hz}$. The corresponding Strouhal number for excitation is $S t_{p}=$ $0.15,0.30,0.55$.

The flow visualization was performed to grasp flow patterns of the excited jet. The jet mean velocity was fixed at $U_{0}=10 \mathrm{~m} / \mathrm{s}$. The corresponding Reynolds number is $R e=6700$. Figures 5(a) to (d) show the photographing images with the each excitation frequency by smoke method. The jet flows from the left to the right. Left images are looked into the vortex structures in the near field of the jet, and the visualization images in the far field are showed in right images. The typical vortex formations in the non-excited jet are shown in Figure 5(a). A shear layer separated from the nozzle lip $(y / w=$ \pm 0.5 ) and rolled up into the vortex at about $x / w=3.5$ with regular spacing. The non-excited vortex shedding frequency was $300 \mathrm{~Hz}$, it corresponds to $f_{0}$. The vortices showed the symmetric alignment which is known as varicose mode. The pair vortex rolled up respect to the jet axis. Then the pair vortex grew up and broke down in the range $3.5<x / w<7$. The vortex merging process and the jet flapping motion were not observed. Vortex formation in the excited jet is displayed in Figures 5(b)-(d). When the jet was excited at $S t_{p}=0.15$, asymmetric alignment of vortices which is known as sinuous mode was clearly observed. The excitation led to the formation switching from the symmetric mode to the asymmetric mode. In this case, it is clearly observed that the jet col- 
(a) $S t_{p}=0.0$



(b) $S t_{p}=0.15$

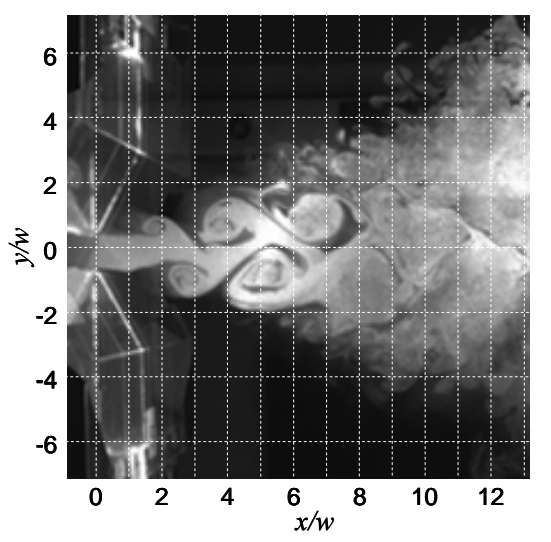

(c) $S t_{p}=0.30$

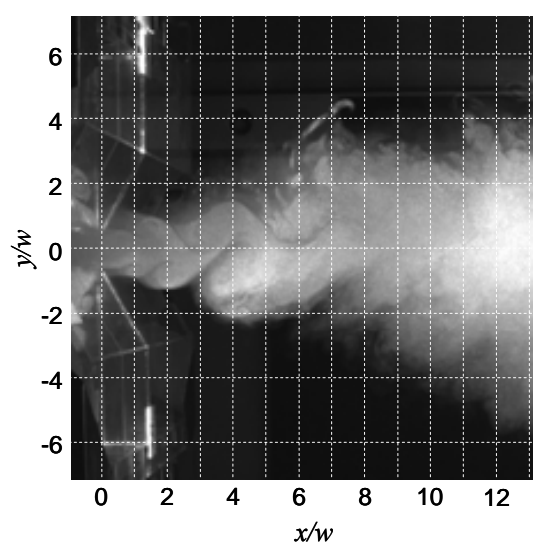

(d) $S t_{p}=0.55$

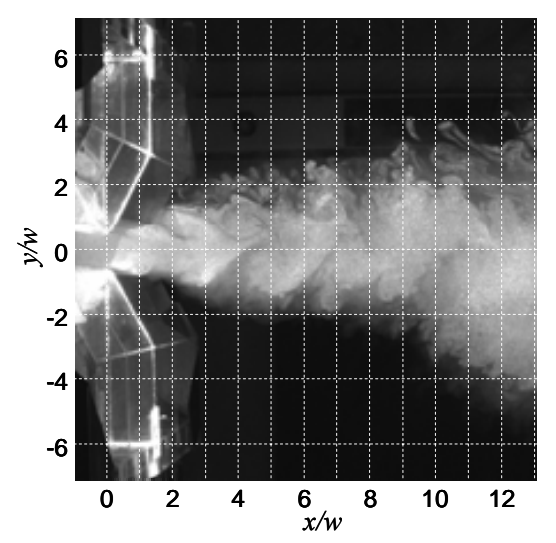




Figure 5. Visualization images $\left(R e=6.7 \times 10^{3}\right.$, Left: enlarged view of right image) 
umn moved laterally. Hence the jet flapping is enhanced with the sub-harmonic and asymmetric excitation. The vortex size is larger than those occurring in the non-excited jet. The roll-up position of vortex was at approximately $x / w=1.5$, and the streamwise interval between the vortices was shorter than that of the non-excited jet.

The vortex could still be observed even at the far downstream region. In the right image, it was found that the jet spreading angle after $x / w>4$ is approximately two times that of the non-excited jet, which implies enhancement of entrainment. For $S t_{p}=0.30$, this Strouhal number is the same as $S t$, the vortex alignment shows sinuous mode, it was close to that for $S t_{p}=0.15$. Although the vortex formation was not clear at this excitation frequency, it was found that the roll-up position of vortex shifts upstream than that for $S t_{p}=0.15$. The streamwise distance between the vortices was diminished, and the jet spreading rate was decreased in comparison with the result for $S t_{p}=0.15$. For $S t_{p}=0.55$ as shown in Figure 5(d), the jet spread rate did not change significantly with the excitation. It was almost the same as that in the non-excited jet. These results show that the sub-harmonic excitation is the most effective frequency on the jet flapping motion.

\subsection{Velocity Measurement}

To investigate the relationship between the velocity distribution and the jet flapping motion, the streamwise mean velocity distribution along the jet axis is measured and shown in Figure 6(a). The potential core region was observed in $x / w<4$ without excitation. The velocity decay started at $x / w=4$, then the velocity decreased monotonically in the downstream region after $x / w=8$. This centerline velocity was proportional to minus one-half power of the $x$. For the excited jet, on the other hand, the potential core region became shorter. For $S t_{p}=0.15$, it was different from the non-excited jet that the velocity decayed slowly from $x / w=10$, then rapid decreasing was observed from $x / w=20$. The jet has the axsymmetric profile because that the centerline velocity was proportional to minus one power of $x$. This result shows that the velocity decay decelerating in the region of $10<x / w<20$ depends on the jet shrinkage to the nozzle spanwise direction. As increasing the forcing Strouhal number, this tendency becomes weak.

Next the distribution of the fluctuating velocity along the jet centerline is plotted in Figure 6(b). Solid marks and open marks show the root mean square value of the periodic velocity, $\tilde{u}_{r m s}$, and the non-periodic velocity, $\hat{u}_{r m s}$, respectively only for the excited jet. The solid line is the turbulence velocity without excitation, $u_{r m s}^{\prime}$, which includes of periodic velocity, since the velocity decomposition could not be applied to the non-excited jet. The value of $\tilde{u}_{r m s}$ grows slowly when the value of $S t_{p}$ is
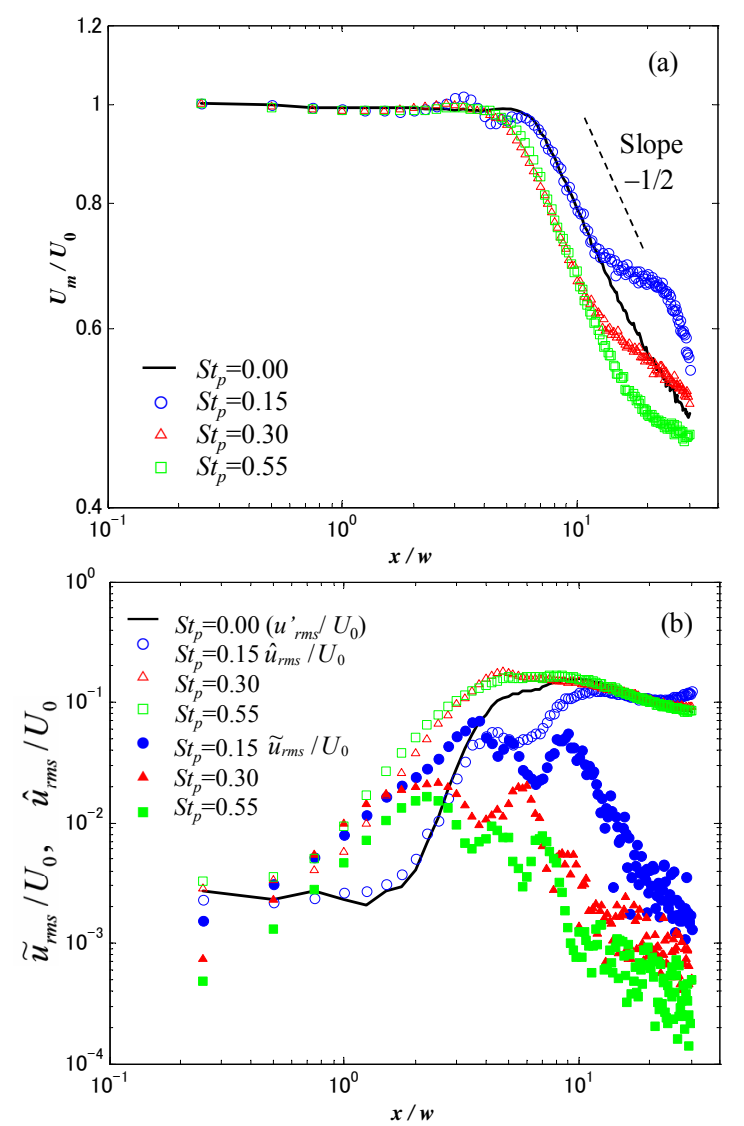

Figure 6. Streamwise velocity distributions $\left(\operatorname{Re}=6.7 \times 10^{3}\right)$.

small, but the peak of $\tilde{u}_{r m s}$ is high. This fact supports the relationship, which was revealed by the flow visualization shown in Figure 5, between the flapping motion and the value of $S t_{p}$. As increasing the value of $S t_{p}$, a 'neck' which is the portion of flapping motion begins to appear shifts upstream, and the flapping amplitude becomes small. For $S t_{p}=0.15$, in the downstream region, the value of $\tilde{u}_{r m s}$ was still high compared with that the other excitation conditions. When the jet is excited with higher value of $S t_{p}$, on the contrast, it is found that the $\tilde{u}_{r m s}$ grows from more upstream region, and the peak value of $\tilde{u}_{r m s}$ becomes smaller. For $S t_{p}=0.15$, the distribution of $\hat{u}_{r m s}$ corresponds that of $u_{r m s}^{\prime}$ in the upstream region of $x / w=3$, but as the value of $S t_{p}$ increases, the value of $\hat{u}_{r m s}$ grows rapidly compared to the nonexcited jet. This tendency is the same as that of $\tilde{u}_{r m s}$. The maximum value of $\hat{u}_{r m s}$ increases with $S t_{p}$.

The contour maps of the mean velocity $U$ and root mean square value of the velocity fluctuation $u_{r m s}^{\prime}$ are shown in Figures 7(a) and (b). The white area in the maps is outside the hot wire measurement. These results indicate only the positive range of $y$, because of that the jet behavior was symmetry respect to the jet centerline. The high velocity regions are observed near the jet exit in Figure 7(a). It is clearly observed that the streamwise- 
length of this region is shortened with the excitation, and the jet spreads wider than that for $S t_{p}=0.00$. These trends were conspicuous in particular for $S t_{p}=0.15$, and were corresponding with the visualization results shown in Figure 5.

In the contour map of the $u_{r m s}^{\prime}$ shown in Figure 7(b), the value of fluctuating velocity is high in the shear layer, the region around $y / w=0.5$. For $S t_{p}=0.3$, this area spreads widely and extends to the downstream direction. For $S t_{p}=0.15$, distribution patterns of the value of $u_{r m s}^{\prime}$ is completely different from the other excitation condi- tions. Additionally it should be noted that a high value area of $u_{r m s}^{\prime}$ was also observed at $x / w=6$ on the jet centerline. This is due to the influence of the large-scale vortex which rolls up in the shear layer reaches to the jet centerline (see Figure 5).

In order to understand the flapping mode oscillation from the instantaneous flow field under the excitation, the contour maps of the velocity $U+\tilde{u}(\theta)$ for $S t_{p}=$ $0.15,0.30,0.55$ are shown in Figures 8(a) to (c). The images were extracted at an interval one-fourth of the excitation period $T$.

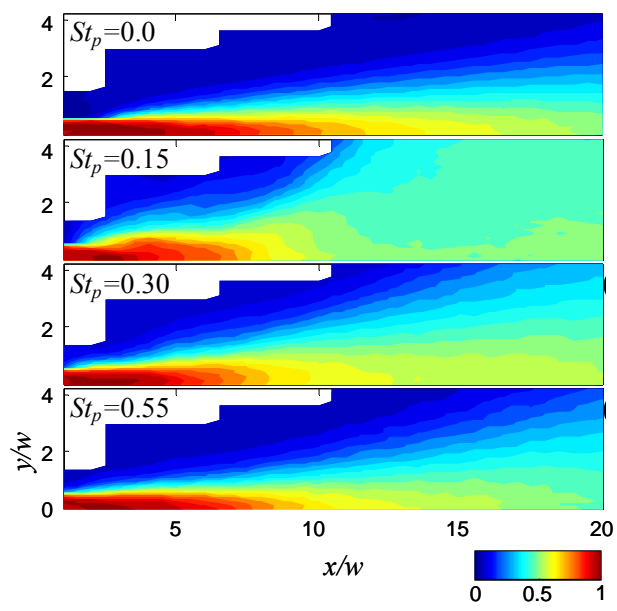

(a) $U / U_{0}$

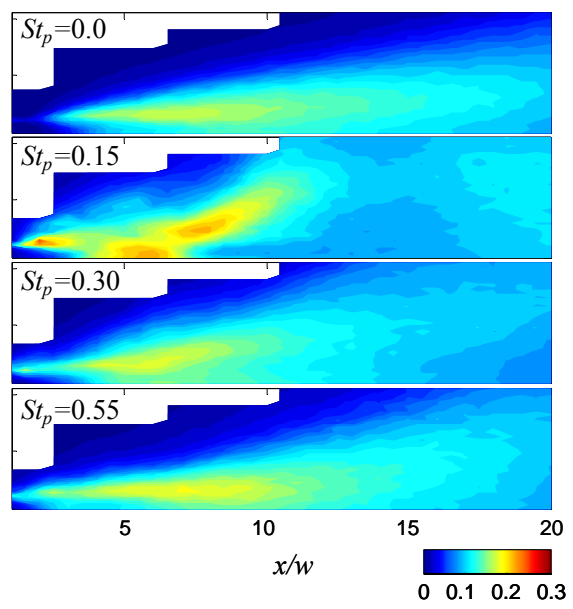

(b) $u_{\mathrm{rms}}^{\prime} / U_{0}$

Figure 7. Velocity contour maps with excitation $\left(R e=6.7 \times 10^{3}\right)$.

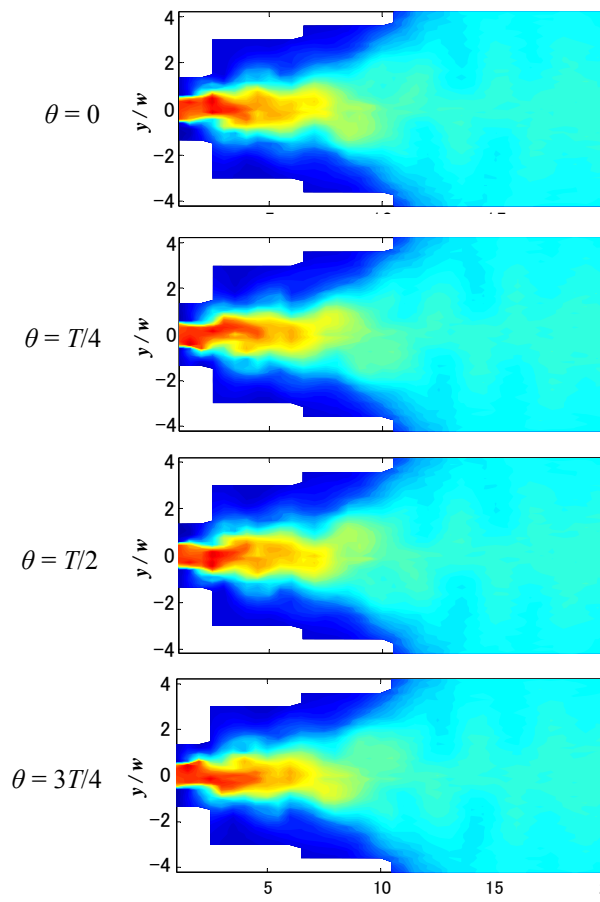

(a) $S t_{p}=0.15$
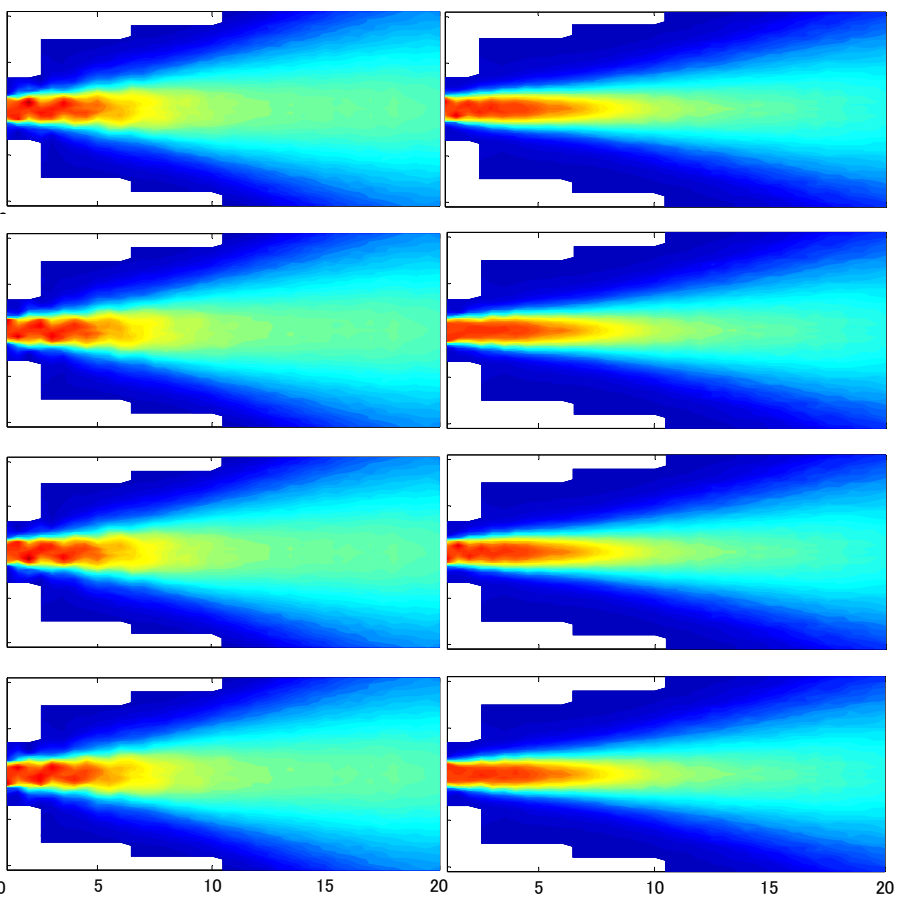

(b) $S t_{p}=0.30$

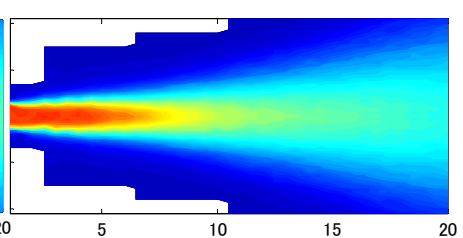

(c) $S t_{p}=0.55$

Figure 8. Velocity contour maps of $U+\tilde{u}(\theta)$ with excitation $\left(\operatorname{Re}=6.7 \times 10^{3}\right)$. 


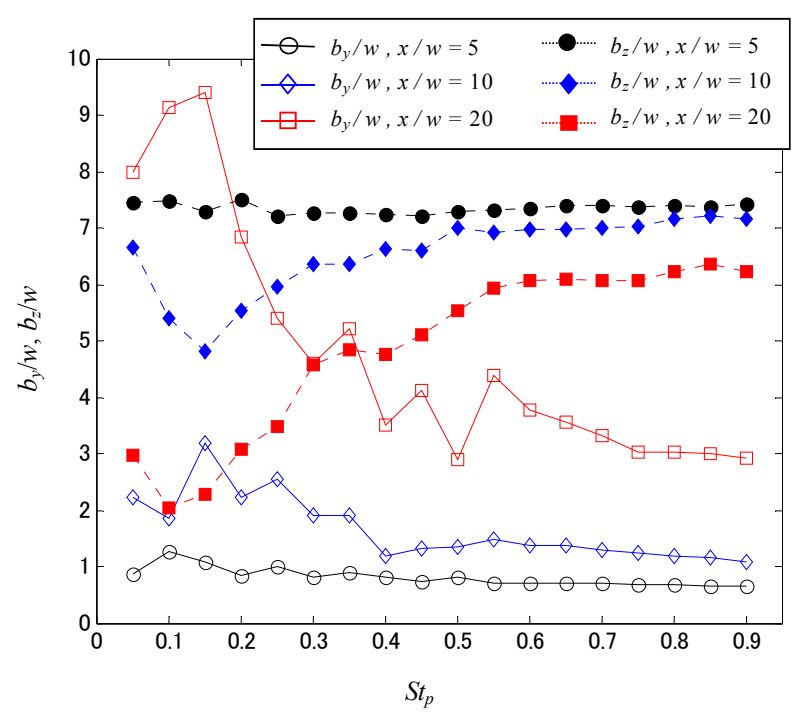

Figure 9. Comparison of jet half width $\left(\operatorname{Re}=6.7 \times 10^{3}\right)$.

Tracking the large-scale cluster of high value of $U+\tilde{u}(\theta)$, it is recognized that the jet flaps laterally in the nozzle width direction for all conditions, and the amplitude of the flapping motion is different with the excitation condition. When the value of $S t_{p}=0.15$, the jet column strongly flapped laterally, and asymmetrically distributed high-velocity region were observed in the downstream. The spread width of the jet was remarkably enhanced in the region of $x / w>7$, associated with the distinctive shedding of the large-scale vortex. For $S t_{p}=$ 0.30 , the jet flapping is also observed up to $x / w=7$, and the amplitude decays along the jet axis. The flapping frequency was synchronized with that of the excitation, thus the wave length of the flapping for $S t_{p}=0.30$ becomes half of that for $S t_{p}=0.15$.

A weak flapping motion (oscillation) was observed for $S t_{p}=0.55$ of $x / w<2$, the oscillation decreased in downstream region as shown in Figure 8(c).

Figure 9 presents distributions of the jet half width plotted against $S t_{p}$ at $x / w=5,10$ and 20. The maximum value of $b_{y} / w$ occurs for $S t_{p}=0.15$ at $x / w=5$ and 10 , then $b_{z} / w$ has the minimum value. Also, in comparison between $b_{y} / w$ and $b_{z} / w$, axis switching occurs in the excitation of $S t_{p}<0.35$ at $x / w=20$. The jet half width of $b_{y} / w$ decreased with increasing of $S t_{p}$.

\section{Conclusion}

This study focused on the development in space of vortex structure evolution in the flapping rectangular jet under asymmetric acoustic excitation condition. The main results of this study are that the excitation frequency has significant effects on the jet flapping. As the excitation frequency is high, the position where the vortex begins to appear becomes upstream, and the streamwise interval between vortices becomes shorten, and then the vortex size becomes small. Then the jet characteristics with higher excitation frequency do not differ from the nonexcited jet. As the excitation frequency decreases, the jet flapping was more clearly observed, and the jet spreads more rapidly. The excitation with sub-harmonic of natural vortex shedding frequency has significant effects on the flapping of the jet.

\section{Acknowledgements}

We would like to thank Tetsuya Inagaki and Yasumitsu Matsubara of graduate students in Shinshu University for their sincere assistance during the preparation of the paper. This work was supported partly by JGC-S SCHOLARSHIP FOUNDATION and Nagano Society for the Promotion of Science.

\section{REFERENCES}

[1] S. C. Crow and F. H. Champagne, "Orderly Structure in Jet Turbulence," Journal of Fluid Mechanics, Vol. 48, No. 3, 1971, pp. 547-591. doi:10.1017/S0022112071001745

[2] G. Brown and A. Roshko, "On Density Effects and large Structure in Turbulent Mixing Layers," Journal of Fluid Mechanics, Vol. 64, No. 4, 1974, pp. 775-816. doi: $10.1017 / \mathrm{S} 002211207400190 \mathrm{X}$

[3] A. K. M. F. Hussain and K. B. M. Q. Zaman, "Vortex Pairing in a Circular Jet under Controlled Excitation, Part 2, Coherent Structure Dynamics," Journal of Fluid Mechanics, Vol. 101, No. 3, 1980, pp. 493-544. doi:10.1017/S0022112080001772

[4] K. Toyoda, T. Okamoto and Y. Shirahama, "Eduction of Vortical Structures by Pressure Measurements in Noncircular Jets," Applied Scientific Research, Vol. 53, No. 3-4, 1994, pp. 237-248. doi:10.1007/BF00849102

[5] F. F. Grinstein, E. J. Gutmark, T. P. Parr, D. M. Hanson-Parr and U. Obeysekare, "Streamwise and Spanwise Vortex Interaction in an Axisymmetric Jet. A Computational and Experimental Study," Physics of Fluids, Vol. 8, No. 6, 1996, pp. 1515-1524. doi:10.1063/1.868927

[6] S. Kida and M. Takaoka, "Bridging in Vortex Reconnection," Physics of Fluids, Vol. 30, No. 10, 1987, pp. 29112914. doi:10.1063/1.866066

[7] S. Iio, K. Takahashi, Y. Haneda and T. Ikeda, "Flow Visualization of Vortex Structure in a Pulsed Rectangular Jet," Journal of Visualization, Vol. 11, No. 2, 2008, pp. 125-132. doi:10.1007/BF03181927

[8] S. Iio, T. Kawamura, M. Matsubara, T. Yoshida and T. Ikeda, "Vortex Behavior of Pulsating Jets from a Rectangular Nozzle,” JSME International Journal Series B, Vol. 49, No. 4, 2006, pp. 988-994.

[9] N. Fujisawa, Y. Takizawa, T. Kohno and S. Tomimatsu, "Active Control of Flow Oscillations in Jet-Wedge System by Acoustic Feedback," Journal of Fluids and Structures, Vol. 19, No. 1, 2004, pp. 111-122. doi:10.1016/j.jfluidstructs.2003.09.001

[10] H. Sato, "The Stability and Transition of a Two-Dimen- 
sional Jet," Journal of Fluid Mechanics, Vol. 7, No. 1, 1960, pp. 53-80. doi:10.1017/S0022112060000049

[11] C. Ho and P. Huerre, "Perturbed Free Shear Layers," Annual Review of Fluid Mechanics, Vol. 16, 1984, pp. 365-422. doi:10.1146/annurev.fl.16.010184.002053

[12] E. Gutmark and C. M. Ho, "Preferred Modes and the Spreading Rates of Jets," Physics of Fluids, Vol. 26, No. 10, 1983, pp. 2932-2938. doi:10.1063/1.864058

[13] V. W. Goldschmidt and P. Bradshaw, "Flapping of a plane jet," Physics of Fluids, Vol. 16, No. 3, 1973, pp. 354-355. doi:10.1063/1.1694348

[14] J. C. de Gortari and V. W. Goldschmidt, "The Apparent Flapping Motion of a Turbulent Plane Jet-Further Experimental Results," Journal of Fluids Engineering, Vol. 103, No. 1, 1981, pp. 119-126. doi:10.1115/1.3240757

[15] O. V. Atassi and R. M. Lueptow, "A Model of Flapping Motion in a Plane Jet," European Journal of MechanicsB/Fluids, Vol. 21, No. 1, 2002, pp. 171-183. doi:10.1016/S0997-7546(01)01176-1

[16] B. L. Smith and A. Glezer, "Jet Vectoring Using Syn-

\section{Nomenclature}

$b_{y}$ : Jet half width in nozzle width direction [m]

$b_{z}$ : Jet half width in nozzle spanwise direction [m]

$f_{0}$ : Natural vortex shedding frequency $[\mathrm{Hz}]$

$S t$ : Strouhal number of unforced jet

$S t_{p}^{*}:$ Strouhal number for forced jet

$T$ : Excitation period [sec.]

$U$ : Mean velocity $[\mathrm{m} / \mathrm{s}]$

$U_{0}$ : Jet mean velocity at the nozzle center $[\mathrm{m} / \mathrm{s}]$

$\hat{u}$ : Non-periodic velocity fluctuation $[\mathrm{m} / \mathrm{s}]$

$\tilde{u}$ : Periodic velocity fluctuation $[\mathrm{m} / \mathrm{s}]$ thetic Jets," Journal of Fluid Mechanics, Vol. 458, 2002, pp. 1-34. doi:10.1017/S0022112001007406

[17] T. Koso and T. Kinoshitat, "Agitated Turbulent Flowfield of a Circular Jet with an Annular Synthetic Jet Actuator," Journal of Fluid Science and Technology, Vol. 3, No. 2, 2008, pp. 323-333. doi:10.1299/jfst.3.323

[18] H. Hasegawa and K. Takahashi, "Experimental Investigation of the Generation of a Longitudinal Vortex in a Transverse Synthetic Jet with Different Excitation Frequencies," Journal of Fluid Science and Technology, Vol. 6, No. 4, 2011, pp. 412-424. doi:10.1299/jfst.6.412

[19] S. Iio, K. Hibino, M. Matsubara and T. Ikeda, "Acoustic Control of an Impinging Planar Jet upon a Wedge," Journal of Fluid Science and Technology, Vol. 3, No. 2, 2008, pp. 274-281. doi:10.1299/jfst.3.274

[20] A. K. M. F. Hussain and W. C. Reynolds, "The MechanIcs of an Organized Wave in Turbulent Shear Flow," Journal of Fluid Mechanics, Vol. 41, No. 2, 1970, pp. 241258. doi:10.1017/S0022112070000605

$u^{\prime}$ : Velocity fluctuation $[\mathrm{m} / \mathrm{s}]$

$u_{p}$ : Velocity fluctuation at slot exit $[\mathrm{m} / \mathrm{s}]$

$w$ : Nozzle width [m]

$\theta$ : particular time [sec.]

\section{Subscript}

$r m s$ : Root mean square value

\section{Superscript}

*: Non-dimensional 